\title{
Tenzing: documenting contributorship using CRediT
}

\author{
Alex O. Holcombe ${ }^{1}$, Marton Kovacs ${ }^{2}$, Frederik Aust ${ }^{3}$, Balazs Aczel ${ }^{2}$ \\ ${ }^{1}$ School of Psychology, University of Sydney, ${ }^{2}$ Institute of Psychology, ELTE, Eotvos \\ Lorand University, Budapest, Hungary, ${ }^{3}$ University of Cologne
}

\section{Abstract}

Scholars traditionally receive career credit for a paper based on where in the author list they appear, but position in an author list often carries little information about what the contribution of each researcher was. "Contributorship" refers to a movement to formally document the nature of each researcher's contribution to a project. We discuss the emerging CRediT standard for documenting contributions and describe a web-based app and $\mathrm{R}$ package called tenzing that is designed to facilitate its use. tenzing can make it easier for researchers on a project to plan and record their planned contributions and to document those contributions in a journal article.

\section{Introduction}

Scholarly journal articles evolved from letters penned by individuals reporting scientific observations or experiment results. These letters listed only a single author, and it was clear that that person (almost always a man) was claiming credit for all aspects of the work reported.

Today, over three hundred years later, most science is done by groups of people, not by lone individuals. Different members of the team usually have different roles. Yet until recently, journals still operated as if there was no need to provide any information other than a list of names - the author list. Some information could be tentatively inferred from the order of names in the list, but how order is determined reflects often-unwritten practices around authorship that can be obscure to people outside a subfield and can differ substantially between labs.

In the presence of uncertainty, people fall back on their priors. This has unfortunate effects for junior authors who do not have many papers to their name: when people see a list of authors with no explicit indication of who did what, they may give an outsize amount of credit to the senior author.

Fortunately, over the last few decades, many journals have begun to encourage, and some to require, that teams give some indication of who did what in the work reported by a paper. In some journals, this is done in a brief "Author Note" or "Author Information" section. Thanks to this development, researchers are more likely to get the specific recognition they deserve. The included information would ideally be utilized by funders of scientists to allocate resources more 
effectively, so that teams with the right combination of skills would more often be supported. Moreover, those who hire scientists, such as universities and research institutes, should be able to assemble more effective teams for particular disciplines and projects.

Unfortunately, these potential benefits have been held back by a lack of standardization. Without a consistent vocabulary for describing what each researcher did in a project, and without a structured format for that information, it is difficult to aggregate across papers the type of contributions a researcher makes. For institutions and funders interested in supporting the right combinations of people, it is difficult to tally the sorts of contributions typically involved in different sorts of projects.

This issue is also faced by other realms, and some have devised solutions. For commercial music, the recording industry uses an International Standard Musical Work Code. This contains metadata for musical works that provide the identities of contributors and indicates whether they served the roles of, for example, composer, lyricist, or arranger (CISAC, 2019; Gilliéron, 2006). A search of the associated ISWC database allows people to find the works that a musician has contributed to and what their role was in each work (http://iswcnet.cisac.org/).

In scientific research, while roles may not be as clear cut as typical in the music industry, there still are useful distinctions that can be made, such as contributions to the analysis of data versus to the drafting of a manuscript, or to the acquisition of data.

In 2014, the first formal taxonomy was developed for scientific research-CRediT, the Contributor Role Taxonomy (Allen et al., 2014). CRediT defines fourteen different types of contributions (https://casrai.org/credit/), and over the last several years, it has been taken up by hundreds of journals (Fennell, 2019) and dozens of publishers (see Adopters at https://casrai.org/credit/) and been endorsed by a number of journal editors (McNutt et al., 2018).

The use of CRediT not only can provide better documentation of the contributions of individual researchers, but also it enables meta-scientific research, such as into the different distribution of contributions indicated for women and men (Macaluso, Larivière, Sugimoto, \& Sugimoto, 2016).

The CRediT standard includes a specification for how to report contributorship information in the metadata that is associated with manuscript webpages (JATS-XML). But many publishers do not yet have the capability to do this. The organizations behind preprint servers are one example-none are currently able to create CRediT metadata in JATS-XML format. In these cases, it can be useful for researchers to publish CrediT information in plain text in their manuscripts. Many journals make no mention of CRediT but ask researchers to indicate what each author did in the "Author Note" or similar section of the manuscript. Researchers can use CRediT to do this, in their preprints and in their submitted manuscripts.

To facilitate researcher reporting of contributorship information in manuscripts and journal articles, we created tenzing, a web app and R package (Kovacs et al., 2020) for researchers and publishers. First, we will review how journals are currently using and report CRediT information. Second, we explain how tenzing can facilitate researcher and journal use of 
CRediT. Finally, we describe broader issues associated with CRediT contributorship that should be addressed as fields move forward with the usage of contributorship.

\section{How journals are using CRediT}

An increasing number of scientific journals offer authors the opportunity to indicate which CRediT category each of an article's authors contributed to. For example, in the submission interface of eLife, authors encounter an array of boxes where they can tick to indicate which category each author contributed to.

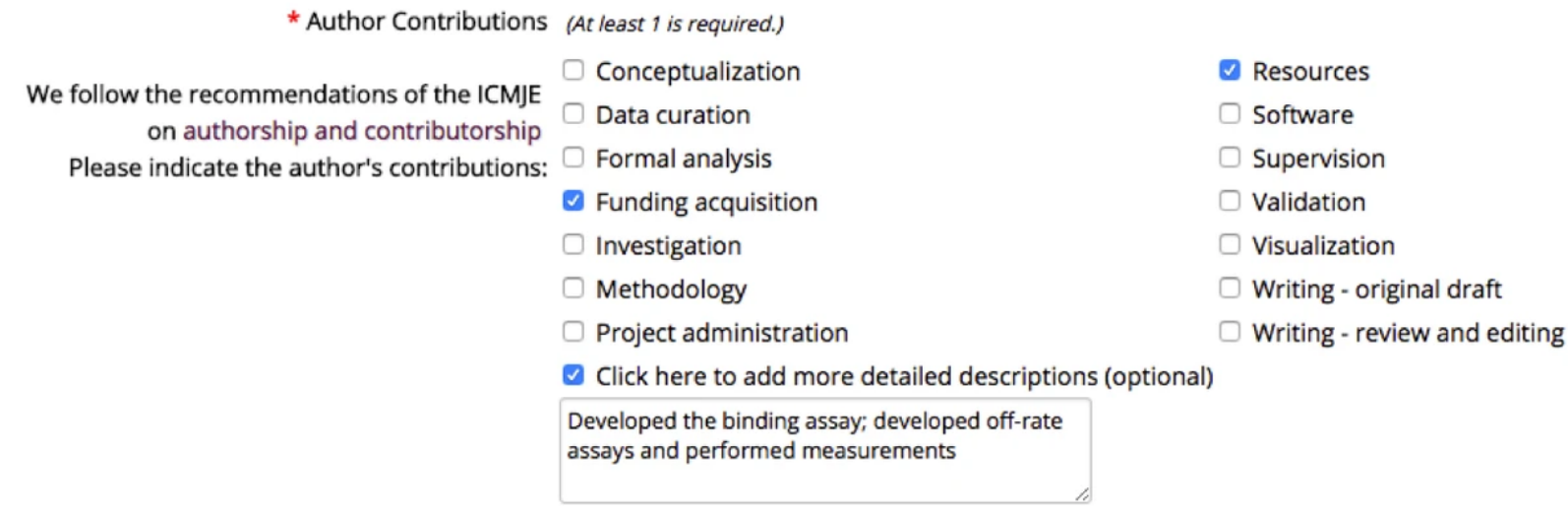

Figure 1. The journal eLife's interface for indicating contributions when submitting a manuscript, available online at https://elifesciences.org/inside-elife/f39cfcf5/enabling-the-contributorroles-taxonomy-for-author-contributions.

PLoS journals provide a similar facility, as do over 1200 Elsevier journals (https://www.elsevier.com/about/press-releases/corporate/elsevier-expands-credit-approach-toauthorship). From various perspectives, however, not considering contributor roles until the time of submission can be problematic.

In science, by the time an author submits a manuscript, the associated research project sometimes was completed months or even years before. Currently, not until the submitting author is confronted with the journal submission page are they likely to communicate with the other authors about what categories they contributed to. At this point, the collective memory of each collaborators' contributions may be fuzzy. Moreover, establishing a consensus between collaborators necessitates interruption of the submission of the manuscript until the submitting author hears from all the other authors and works to resolve any disagreement about various contributions, such as who contributed to the original draft of the manuscript.

There is reason to believe that, when not discussed until after project completion, the rate of disagreements regarding author contributions may be high. Research integrity offices at universities report that the majority of the cases they see are disagreements about authorship. In part for that reason, many recommend that researchers discuss authorship at the beginning of a project. This may be even more important when the manuscript is to provide not only a list of author names, but also a specification of each author's contributions. 
Most authorship disputes are settled informally, but still may leave some people bitter at being excluded, or resentful that some people were included on an authorship list without any evidence they deserved it. The same likely applies to disputes over which contribution categories a researcher contributed to. It is probably best to get some agreement on these at the beginning of a project, so that researchers can proceed with some confidence around both what they are expected to do and what kind of credit they will get for it.

In some fields, the submitting author is often the most junior author. This may often be the case when a PhD student submits her first paper, for example. A student is probably not in the best position to arbitrate disputes or push back on project contributors who may be overclaiming regarding their contribution. This is an additional reason to separate the documentation of contributorship from the submission tasks that the first author typically completes.

\section{What tenzing does}

tenzing (https://martonbalazskovacs.shinyapps.io/tenzing/) is a web app and associated $\mathrm{R}$ package that allows researchers to record contributorship information at any time, for eventual provision to a journal. The app is named after the mountaineer Tenzing Norgay, who together with Edmund Hillary was the first to reach the summit of Mount Everest. Norgay arguably received less credit than was appropriate given his contribution.

Here we will describe the use of tenzing solely in terms of the web app (https://martonbalazskovacs.shinyapps.io/tenzing/), although one can also use it via the underlying R package (https://github.com/marton-balazs-kovacs/tenzing)_full documentation for tenzing can be found at https://marton-balazs-kovacs.github.io/tenzing/

Use of tenzing starts with a spreadsheet template (provided as a Google Sheet, http://bit.ly/tenzingTemplate, but one can also use it in Excel). Researchers make their own copy of the template and then, in the rows, enter the names of their collaborators on a project. One column is dedicated to each of the fourteen CRediT categories, to be checked off to indicate which categories each researcher contributed to. Because some CRediT categories are not entirely self-explanatory, one can hover the cursor over the column names to see some additional defining information.

\begin{tabular}{|c|c|c|c|c|c|c|c|c|c|c|}
\hline $\begin{array}{l}\text { Order in } \\
\text { publication }\end{array}$ & Firstname & Middle name & Surname & $\begin{array}{l}\text { Conceptu } \\
\text { alization }\end{array}$ & $\begin{array}{l}\text { Data } \\
\text { Curation }\end{array}$ & $\begin{array}{l}\text { Formal } \\
\text { Analysis }\end{array}$ & $\begin{array}{l}\text { Funding } \\
\text { Acquisition }\end{array}$ & $\begin{array}{l}\text { Investi } \\
\text { gation }\end{array}$ & $\begin{array}{l}\text { Metho } \\
\text { dology }\end{array}$ & $\begin{array}{l}\text { Projec } \\
\text { Admir } \\
\text { on }\end{array}$ \\
\hline 1 & Alex & 0 & Holcombe & $\checkmark$ & $\square$ & $\square$ & $\square$ & $\square$ & $\square$ & L \\
\hline 2 & Marton & & Kovacs & $\checkmark$ & $\square$ & $\square$ & $\square$ & $\square$ & $\square$ & {[} \\
\hline 3 & Frederik & & Aust & $\square$ & $\square$ & $\square$ & $\square$ & $\square$ & $\square$ & \\
\hline 4 & Balazs & & Aczel & $\bar{v}$ & $\square$ & $\square$ & $\square$ & $\square$ & $\square$ & \\
\hline
\end{tabular}

Figure 2. Partial screenshot of the spreadsheet template (http://bit.ly/tenzingTemplate)

Around the time of the start of a project, a lead researcher may choose to send the link to the Sheet to all those involved, who can then indicate the areas they plan to contribute to. At the end of the project, or when plans change during the project, this Sheet can be revisited. 
When the researchers are ready to submit to a journal, they upload their filled-out spreadsheet to the tenzing app. They can then click a button to generate any of various outputs. A first output is unrelated to CRediT: a list of the authors' names, with annotations indicating the institutions they are affiliated with, formatted to be suitable to paste into the title page of a manuscript file (Figure 3).

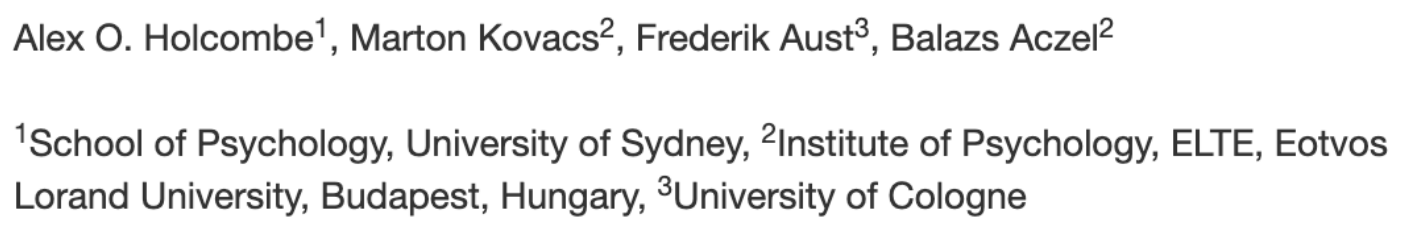

Figure 3. A screenshot of the author list and affiliation output screen.

For CRediT, tenzing also outputs a brief report in the form of a list indicating which contributor did what (Figure 4). This can be pasted into the section of a manuscript known at some journals as the Author Note. It is particularly appropriate for journals whose publishing platform does not support the machine-readable CRediT metadata. Collabra: Psychology is a journal that encourages researchers to provide CRediT information in the Author Contributions section, although their publisher has not yet implemented creation of CRediT metadata in the article contents.

\section{Author contributions}

Conceptualization: Alex O. Holcombe, Marton Kovacs and Balazs Aczel. Software: Alex O. Holcombe, Marton Kovacs, Frederik Aust and Balazs Aczel. Writing - Original Draft Preparation: Alex O. Holcombe, Marton Kovacs, Frederik Aust and Balazs Aczel.

Writing - Review \& Editing: Alex O. Holcombe, Marton Kovacs, Frederik Aust and Balazs Aczel.

\section{Copy output to clipboard}

$$
\downarrow \text { Download file }
$$

Close

Figure 4. Screenshot of the tenzing window that provides a report of author contributions

The publishing platforms used by dozens of publishers can include CRediT metadata in JATSXML-format in the journal article webpages (see list of "adopters" at https://casrai.org/credit/). tenzing can generate this JATS-XML information itself for users to download (Figure 5). Ideally, researchers would be able to upload this to a journal submission portal when submitting their 
manuscript. Unfortunately, at present no journals appear to be capable of this, although a few publishers have indicated to us that they're interested in changing their systems to accommodate this.

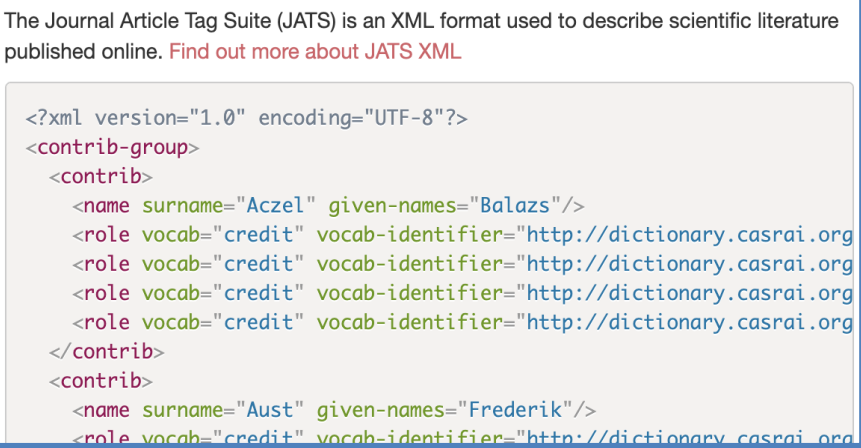

Figure 5. A screenshot of a portion of the JATS-XML output provided by tenzing.

Some researchers write manuscripts in R Markdown and use the papaja package (Aust \& Barth, 2020; https://crsh.github.io/papaja man/) to generate manuscripts in APA format for submission to a journal. tenzing generates author metadata in YAML-format, which can be included in the $R$ Markdown file. papaja then includes the CRediT information in the Author Note section of the APA-formatted manuscript.

\section{Tenzing Documenting contributorship with CRediT}

\section{Create your infosheet}

First copy and then fill out this infosheet template

\section{Upload your infosheet}

Browse..

No fille selected

Show infosheet

\section{Download the output}

Show author contributions text

Show author list with affiliations

Show XML file (for publisher use)

Show papaja YAML

\section{How to use the application}

1. Create your infosheet

- Copy the infosheet template in your Google Drive File -> Make a copy

- Fill out your copy of the infosheet

- You can share it with your collaborators to make the process faster

2. Upload your infosheet

- Download the filled out infosheet to your computer in a .csv, .tsv or .xlsx format

- If you use .xlsx format the contributorship information should be on the first sheet

- Click the "Browse" button and find your infosheet on your computer

- If you want to take a look at the uploaded infosheet click "Show infosheet"

\section{Download the output}

- You can generate 3 types of outputs:

- A human-readable report of the contributions with the "Author Contributions text"

- The contributors affiliation page information for the manuscript with the "Annotated author list with affiliations"

- JATS XML containing the contributions with the "XML (for publishers only)

- papaja compatible YAML code of the contributor roles 
Figure 6. A screenshot of the tenzing app. The bottom portion of both sides describes the four outputs that tenzing provides.

\section{The future of CRediT}

The CRediT standard was primarily designed to allow researchers to indicate what type of contribution they made. However, it also has a facility that allows one to indicate the degree of contribution. Specifically, one can optionally indicate whether each contributor to a particular category played a "lead", "equal", or "supporting" role in the associated work. However, it appears that most journals that use CRediT have opted not to use this feature. Editorial Manager, a journal platform used by thousands of journals, has integrated the degree of contribution feature but as a specific configuration, and most journals using Editorial Manager currently do not appear to have activated it.

An unresolved issue with the degree of contribution facet of CRediT is how it should be used. It seems likely that where "equal" label is used, it must be used for multiple co-authors rather than just one. This is not currently addressed, however, by the CRediT documentation, nor are other possible constraints such as whether "equal" can be used as an intermediate indicator in cases where there are already authors with the "lead" and "supporting" labels. In addition, there is no indication to publishers of how they should indicate degrees of contribution in the machinereadable JATS-XML associated with journal articles, although Aries Systems, the creator of Editorial Manager, has done this by using the "specific-use" attribute (Caroline Webber, personal communication, 8 July 2020).

The degree of contribution issue will likely be addressed by a group convened by the American National Information Standards Organization that is working to formalize CRediT as an ANSI/NISO standard (https://niso.org/press-releases/2020/04/niso-launches-work-contributorrole-taxonomy-credit-initiative). For now, we have chosen to not yet implement the degree of contribution feature in tenzing.

\section{The future of contributorship}

The number of contributors to the average scientific paper has steadily increased over the last several decades (Regalado, 1995; Fanelli \& Larivière, 2016). In part, this has occurred because as knowledge in an area increases, specialization facilitates further advances. Some forms of research today, such as systematic reviews and meta-analyses, are based on bringing together large amounts of evidence from the literature. Library professionals contribute to some such projects with sophisticated searches of papers and databases. For other projects, technicians provide invaluable guidance regarding equipment, programmers create needed software, statisticians provide statistical advice, and informaticists create visualizations or collate information from databases. With science increasingly depending on these tasks getting done, funders need to be able to assess what sorts of projects have most benefited from specialists in order to resource science most effectively. However, people in these specialist roles are often 
not included in author lists, making it difficult to determine the number of specialists contributing to various projects.

One obstacle to greater inclusion of specialist contributors is the current state of journal authorship guidelines. The authorship guidelines for thousands of journals are based on the International Committee of Medical Journal Editors. These guidelines stipulate that only those who contribute to the writing or revising of a manuscript are eligible for authorship (International Committee of Medical Journal Editors, 2018). Journals should consider expanding authorship eligibility, for example by adopting the proposal of McNutt et al. (2018) to eliminate the writing requirement and endorse the use of CRediT (Holcombe, 2019).

CRediT is not a good fit for all disciplines or even all projects within a discipline ( https://blogs.Ise.ac.uk/impactofsocialsciences/2020/01/20/credit-check-should-we-welcometools-to-differentiate-the-contributions-made-to-academic-papers/). An ontology of roles that is both broader than those of CRediT and also more specific has been developed by the National Center for Data to Health, an initiative of the National Center for Advancing Translational Sciences (NCATS) at the National Institutes of Health (Vasilevsky et al., 2020). The scheme is called the Contributor Role Ontology (CRO, https://data2health.github.io/contributor-roleontology/), and it extends the CRediT ontology to include more than fifty roles, including "specimen collection", "librarian", "community engagement", "coordination", and "software testing” (https://www.force11.org/blog/introducing-contribution-role-ontology-developingsustainable-community-driven-approach; Vasilevsky et al., 2020; llik et al., 2018). Given the adoption of CRediT that has already occurred, we anticipate that improvements will occur via extensions or generalizations such as $\mathrm{CRO}$.

CRO has been integrated with Manubot

(https://github.com/data2health/contributorship/issues/5), which creates formatted scientific manuscripts from Markdown. Like papaja, Manubot takes YAML-formatted text that a user has entered into Github regarding CRO roles and integrates it into the resulting manuscript. The CRO scheme could be integrated into tenzing in the future.

If author contributions to a journal's articles are explicitly indicated by a contributorship taxonomy such as CRediT or CRO, how should one think about the order of authorship? One might expect order to still be used for communicating the relative amount that different authors contributed, despite its limitations due to ambiguity around interpreting the meaning of first author and last author in different fields and cultures. However, note that CRediT also allows an indication of degree of contribution, beyond just how many categories a researcher contributed to. Specifically, where multiple individuals serve in the same role, the degree of contribution can optionally be specified as 'lead', 'equal', or 'supporting'.

Deciding on order of authorship may get more and more difficult as the number of authors increases. Having a discussion among the researchers to decide this, without a clear decision process, may be unwieldy. Some have suggested a points system for different types of contributions. The American Psychological Association online authorship resources site for several years has included an example "scorecard" that assigns different types of contributions different numbers of points ( https://www.apa.org/science/leadership/students/authorship- 
determination-scorecard.pdf). For CRediT, one such points system has been created by Mojtaba Soltanlou (https://osf.io/zd84r/). However, the relative value of different sorts of contributions likely differs across projects.

A critically important document for communicating contributions to scholarship is the CV. Traditionally, the extent of different authors' contributions is communicated entirely by the order of authorship. In the future, however, we anticipate that funders or individual researchers will move to CVs that communicate the nature of the contributions made to each journal article. The Rescognito site (Wynne, 2019) has created experimental visualizations https://rescognito.com/0000-0002-9217-0407?v=1, as did Ebersole, Adie, \& Cook in a SIPS hackathon (Holcombe, Vazire, Chartier, et al., 2019), who created a bar graph indicating, for each CRediT category, how many papers a researcher contributed to.

Another piece of infrastructure already supporting CRediT usage is the ORCiD database and metadata for identifying researchers and linking them to their papers and other scholarly contributions (Haak et al., 2012). Usage has grown rapidly, with this year (2020) seeing it grow to over 7,000 papers a month indexed in Crossref, because at least one author used ORCiD (https://www.linkedin.com/pulse/who-uses-orcid-ids-anyway-richard-wynne/). The ORCiD registry includes CRediT information.

With adoption of CRediT growing rapidly, it is becoming more urgent to attend to any problems being encountered in its use or with the standard itself. The NISO effort to formalize CRediT will include a solicitation of feedback, which will be an important opportunity for the scholarly community to shape how contributorship information is recorded. We hope that the usage of CRediT facilitated by tenzing during the feedback period will result in a greater understanding of what about CRediT should be prioritized for refinement or change.

\section{Author Contributions}

Conceptualization: Alex O. Holcombe, Marton Kovacs and Balazs Aczel.

Software: Marton Kovacs, Frederik Aust, Alex O. Holcombe and Balazs Aczel.

Writing - Original Draft Preparation: Alex O. Holcombe, Marton Kovacs, Frederik Aust and Balazs Aczel.

Writing - Review \& Editing: Alex O. Holcombe, Marton Kovacs, Frederik Aust and Balazs Aczel.

\section{Acknowledgments}

We thank the Society for the Improvement of Psychological Science (SIPS) and the participants in the 2019 SIPS Hackathon (Holcombe et al., 2019) on contributorship for discussion. 


\section{References}

Allen, L., Scott, J., Brand, A., Hlava, M., \& Altman, M. (2014). Publishing: Credit where credit is due. Nature News, 508(7496), 312.

Aust, F., \& Barth, M. (2020). papaja: Create APA manuscripts with R Markdown. Retrieved from https://github. com/crsh/papaja

Brand, A.; Allen, L.; Altman, M.; Hlava, M.; Scott, J. (2015). Beyond authorship: Attribution, contribution, collaboration, and credit. Learned Publishing, 28, 151-155.

CISAC (4 February, 2019). CISAC launches major project to upgrade the international musical work identifier. Retrieved June 29, 2020, from https://www.cisac.org/Newsroom/NewsReleases/CISAC-launches-major-project-to-upgrade-the-international-musical-work-identifier

Fanelli, D., \& Larivière, V. (2016). Researchers' Individual Publication Rate Has Not Increased in a Century. PLOS ONE, 11(3), e0149504. https://doi.org/10.1371/journal.pone.0149504

Fennell, C. (2019, 5 July). Fully agree, Elsevier is offering CRediT for close to 200 journals now, with great response from authors \& editors. We're busy getting ready to expand thanks to @AriesMarketing making CRediT even easier for authors \& editors. [Tweet]. Retrieved from https://twitter.com/CatrionaFennell/status/1147119169831350272.

Fotion, N. \& Conrad, C. (1984) Authorship and other credits. Annals of Internal Medicine. 100: 592-594.

Gilliéron, P. (2006). Performing Rights Societies And The Digital Environment. bepress Legal Series, 1436.

Haak, L. L., Fenner, M., Paglione, L., Pentz, E., \& Ratner, H. (2012). ORCID: a system to uniquely identify researchers. Learned Publishing, 25(4), 259-264.

Holcombe, A.O., Vazire, S., Chartier, Ebersole, C., Giner-Sorolla, R., Haroz, S., Moreau, D., Primbs, M., Ling, M., Werner, K., Schnyder, N., Adie, J., Crook, Z., Smout, C., Ribeiro, G, Tangen, J., Aczel, B., Thibault, R., Searston, R., Van 't Veer, A., Schmalz, X. (2019). [Conference session]. Replace journals' writing-based authorship guidelines with a contributorship model. 2019 Annual Meeting of the Society for the Improvement of Psychological Science.

llik, V., Conlon, M., Triggs, G., White, M., Javed, M., Brush, M., Gutzman, K., Essaid, S., Friedman, P., Porter, S., Szomszor, M., Haendel, M. A., Eichmann, D., \& Holmes, K. L. (2018). OpenVIVO: Transparency in Scholarship. Frontiers in Research Metrics and Analytics, 2. https://doi.org/10.3389/frma.2017.00012

International Committee of Medical Journal Editors. Defining the Role of Authors and Contributors. Updated December 2018. Available online: http://www.icmje.org/icmjerecommendations.pdf (accessed on 20 June 2019). 
Kovacs, M., Aust, F., Holcombe, A. O., \& Aczel, B. (2020). tenzing: Documenting contributions to scientific scholarly output with CRediT. R package version 0.1.0. Retrieved from

https://github.com/marton-balazs-kovacs/tenzing

Macaluso, B., Larivière, V., Sugimoto, T., \& Sugimoto, C. R. (2016). Is Science Built on the Shoulders of Women? A Study of Gender Differences in Contributorship: Academic Medicine, 91(8), 1136-1142. https://doi.org/10.1097/ACM.0000000000001261

Macaulcy, D. (1992) Cite the workers. British Medical Journal 305: 120 https://www.bmj.com/content/305/6845/120.1

McNutt, M. K., Bradford, M., Drazen, J. M., Hanson, B., Howard, B., Jamieson, K. H., Kiermer, V., Marcus, E., Pope, B. K., \& Schekman, R. (2018). Transparency in authors' contributions and responsibilities to promote integrity in scientific publication. Proceedings of the National Academy of Sciences, 115(11), 2557-2560.

Regalado, A. (1995). Multiauthor papers on the rise. Science, 268 (5207) (1995), p. 25.

Resnik, D.B. A proposal for a new system of credit allocation in science. Sci. Eng. Ethics 1997, 3, 237-243.

Vasilevsky, N. A., Hosseini, M., Teplitzky, S., Ilik, V., Mohammadi, E., Schneider, J., Kern, B., Colomb, J., Edmunds, S. C., Gutzman, K., Himmelstein, D. S., White, M., Smith, B., O'Keefe, L., Haendel, M., \& Holmes, K. L. (2020). Is authorship sufficient for today's collaborative research? A call for contributor roles. Accountability in Research, 1-21.

https://doi.org/10.1080/08989621.2020.1779591

Wynne, R.C.D. (2019). "Got a DOI? Claim and Give Some CRediT" https://figshare.com/articles/Got a DOI Claim and Give Some CRediT /9733595/1 